\title{
A Morphological and Genetic Mapping Study of Bald Colony Mutants of Streptomyces coelicolor
}

\author{
By M. J. MERRICK* \\ John Innes Institute, Colney Lane, Norwich $\mathrm{NR}_{4}{ }_{7} \mathrm{UH}$
}

(Received 8 April I976)

SUMMARY

\begin{abstract}
Twelve bld mutations of Streptomyces coelicolor resulting in a lack of visible aerial mycelium were mapped genetically. The mutants were classified into three groups on the basis of colony morphology, production of antibiotics and morphology on different carbon sources. Four map locations were found for the bld genes and three of these were very near the loci of whi genes, which are also involved in differentiation. Closely linked bld mutations had similar phenotypes.
\end{abstract}

\section{INTRODUCTION}

Chater (1972) studied 50 whi mutants of Streptomyces coelicolor which were defective at various stages in aerial mycelium development. These mutants fell into six phenotypic classes and the mutations were mapped at eight loci. A further class of developmental mutants includes those in which a mutation prevents any aerial mycelium production. These mutants have been termed bald (bld) and one such mutation, bldAr (s48), has been mapped (Hopwood, 1967). Approximate map locations for some other bld mutations have also been reported (Chater \& Hopwood, 1973; Hopwood et al., 1973) but these mutations were not described in detail. This paper describes genetic mapping studies with a number of bld mutants and their classification using various criteria. A preliminary report of some of this work has been published (Chater \& Merrick, I976).

\section{METHODS}

Organisms. All strains were ultimately derived from the wild-type Streptomyces coelicolor strain A3(2) (Hopwood, 1959). All bld mutants were isolated by visual selection following treatment with either $N$-methyl- $N^{\prime}$-nitro- $N$-nitrosoguanidine (Delić, Hopwood \& Friend, 1970) or 8-methoxypsoralen plus near-ultraviolet irradiation (Townsend, Wright \& Hopwood, I97I). With the exception of bldAI and bldD53, the mutations were isolated in strain $\mathrm{J}_{650}$. All mutations in $\mathrm{J} 650$ were isolated at $35^{\circ} \mathrm{C}$; after purification, their morphology was examined at $25{ }^{\circ} \mathrm{C}$ for temperature-sensitivity. The genetically marked strains used for mapping are listed in Table $\mathrm{I}$. The locations of all the markers on the circular linkage map are shown in Fig. I and the genetic symbols are as listed by Hopwood et al. (I973).

Media and general methods of culture and genetic analysis. Minimal (MM) and complete (CM) agar media, supplements and general methods of culture were as described by Hopwood (1967). All carbon sources were used at I \% (w/v) final concentration. Unless otherwise stated, incubation was at $30^{\circ} \mathrm{C}$.

\footnotetext{
* Present address: A.R.C. Unit of Nitrogen Fixation, University of Sussex, Brighton BNI 9QJ.
} 
Table I. Strains of Streptomyces coelicolor A3(2)

Explanations of symbols are given by Hopwood et al. (1973). Fertility designations are those of Hopwood \& Wright (1976a) and morphological phenotypes of whi mutations are given by Chater (1972).

\begin{tabular}{|c|c|c|}
\hline Strain no. & Genotype & Fertility type \\
\hline 285 & $\operatorname{ade} A 3 \arg A I$ cysB6 strAI uraAI & n.t. \\
\hline 948 & ammAS hisAI serAI & NF \\
\hline 949 & $\arg A I$ cys $A I 5$ nic $A I$ proAI uraAI tps-30 & NF \\
\hline I 190 & hisAI uraAI strAI & $\mathrm{SCPI}^{-}$ \\
\hline 1514 & agaAI $\arg A I$ cysAI 5 nicAI proAI uraAI & NF \\
\hline A200 & adeCvro pheAr strAs & $\mathrm{SCPI}^{-}$ \\
\hline JI92 & cysDI8 leuB5 strAI & NF \\
\hline $\mathrm{J} 200$ & nic $A$ I pheAI strAI whiBaI 8 & NF \\
\hline 3650 & agaAI cys DI8 mthB2 & $\mathrm{NF}$ \\
\hline \multicolumn{3}{|l|}{ whi mutants } \\
\hline JI2 & hisAI strAI whiA72 & NF \\
\hline $\mathrm{J} 49$ & leuBs strAI whiHII 9 & NF \\
\hline $\mathrm{J} 86$ & leuB5 strAI whili 7 & NF \\
\hline J93 & $\arg A T$ strAr whiA72 & n.t. \\
\hline $\mathrm{J} 95$ & cysDI8 strAI whiHII9 & NF \\
\hline JII 8 & cysDI8 strAI whiG7I & NF \\
\hline JII9 & leuB5 strAI whiG7I & NF \\
\hline J127 & cysDI8 strAI whilr 7 & NF \\
\hline \multicolumn{3}{|l|}{ bld mutants } \\
\hline 1169 & bldD53 his $A r$ mthBz pheAI strAI & $\mathrm{NF}$ \\
\hline $\mathrm{J} 660$ & agaAI bldCI 8 cys DI 8 mthB2 & NF \\
\hline J668 & agaAI bldA39 cysDI8 mthB2 & NF \\
\hline J669 & agaAI bldB43 cys DI8 mthB2 & NF \\
\hline $\mathbf{J} 702$ & agaAr bldA39 mthB2 & NF \\
\hline $\mathrm{J} 707$ & agaAI bldA35 mthB2 & NF \\
\hline $\mathrm{J} 708$ & agaAI bldAI6 mthB2 & NF \\
\hline J714 & agaAI bldB43 leuB5 strAI & NF \\
\hline $\mathrm{J} 733$ & agaAI bldA35 cysAIs hisAI uraAI & NF \\
\hline $\mathrm{J} 74 \mathrm{I}$ & agaAI bldCr 8 mthB2 & $\mathrm{NF}$ \\
\hline $\mathrm{J} 777$ & $\arg A I$ bldD 53 strAI uraAI & $\mathrm{NF}$ \\
\hline & n.t., not tested. & \\
\hline
\end{tabular}

Antibiotic production. Production of antibiotics was assessed by growing a confluent lawn of the strain to be tested on CM for 3 days. A $16 \mathrm{~mm}$ diameter plug was then cut from this lawn using a sterile cork-borer. For tests against eubacteria, the plug was surrounded by Difco nutrient agar containing the assay organism at a concentration of about $10^{8}$ cells $\mathrm{ml}^{-1}$ and the plate was incubated overnight at $37^{\circ} \mathrm{C}$. When testing for antibiotic activity against strains of $S$. coelicolor, the plug was placed on a freshly spread lawn of spores of the assay strain on CM and the plate was incubated for 3 days.

Microscopy. Impression preparations were made and examined by the method of Chater (1972).

\section{RESULTS}

\section{Colony morphology of bald mutants}

Colonies were grown for 5 days on MM with glucose as the carbon source. Under these conditions the colony morphology of each strain was found to be stable and reproducible and the mutants could be divided into three distinct morphological classes. None of the mutations produced a temperature-sensitive bld phenotype.

Class I. Mutants in this class had non-pigmented colonies which were smooth after 2 or 


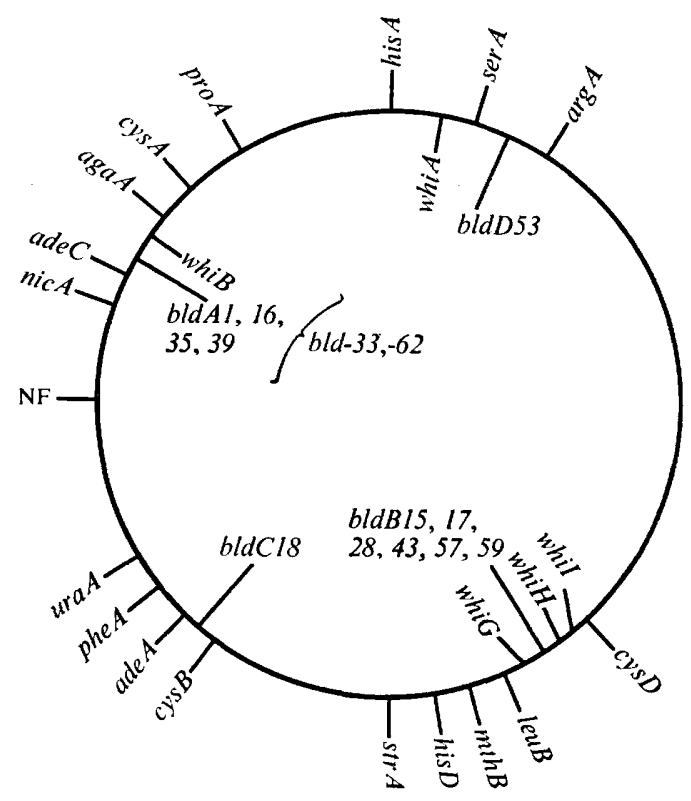

Fig. 1. Linkage map of markers employed. The map and gene symbols are based on those of Hopwood et al. (1973). Morphological markers are given inside the circle, with the allele numbers of the mutations at each locus.

3 days' growth but had a characteristic sculptured pattern on the colony surface after 5 or 6 days (Fig. 2). The colonies were very soft and could easily be broken up with a fine wire. Such mutants replicated well by the normal velvet replica-plating technique. Colony diameter was similar to or sometimes slightly greater than that of wild-type colonies grown under the same conditions. In impression preparations, the hyphae frequently appeared to be mis-shapen, with numerous septa (Fig. 3).

Class 2. These mutants had non-pigmented colonies which were smooth and rather flat with a feathery outline (Fig. 2). Their morphology did not change with age. The colonies were relatively hard, difficult to break up with a wire and replicated poorly. Colony diameter was similar to that of the wild type.

Class 3. Only one mutant (bld-18) was identified in this class. The colonies were smooth with a well defined border (Fig. 2). Young colonies were non-pigmented but often turned pink or red on ageing. They were relatively soft and replicated quite easily. Colony diameter was smaller than that of the wild type.

None of the three classes of mutant produced the earthy odour characteristic of Streptomyces spp.

\section{Phenotypes of bld mutants on different carbon sources}

The parent strain (J650) grew well and had normal colony morphology on arabinose, galactose, glucose, mannose, cellobiose, maltose, glycerol and mannitol. All the bld mutants could also utilize these carbon sources.

The morphology of the bld mutants was examined, using a binocular microscope and by impression preparations, after 5 days' growth on MM supplemented with each of the above carbon sources.

Class I mutants were bald on glucose, mannose and cellobiose but on the other five 

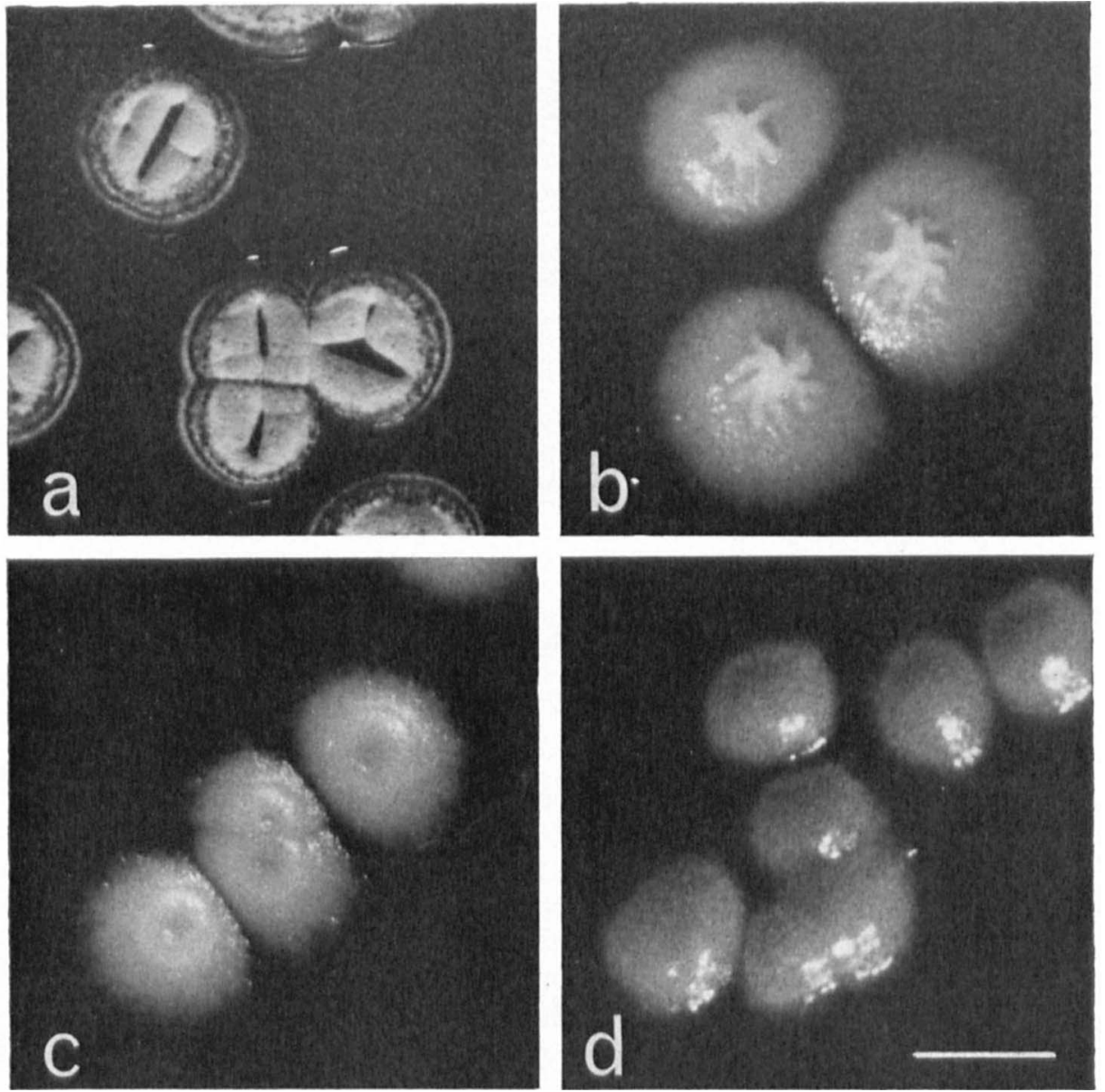

Fig. 2. Colony morphology of bld mutants and wild type: (a) wild type; (b) class I mutant (bldA39); (c) class 2 mutant (bldB43); (d) class 3 mutant (bldCI8). Colonies were grown on MM at $30^{\circ} \mathrm{C}$ for 5 days. Bar marker represents $0.25 \mathrm{~cm}$.
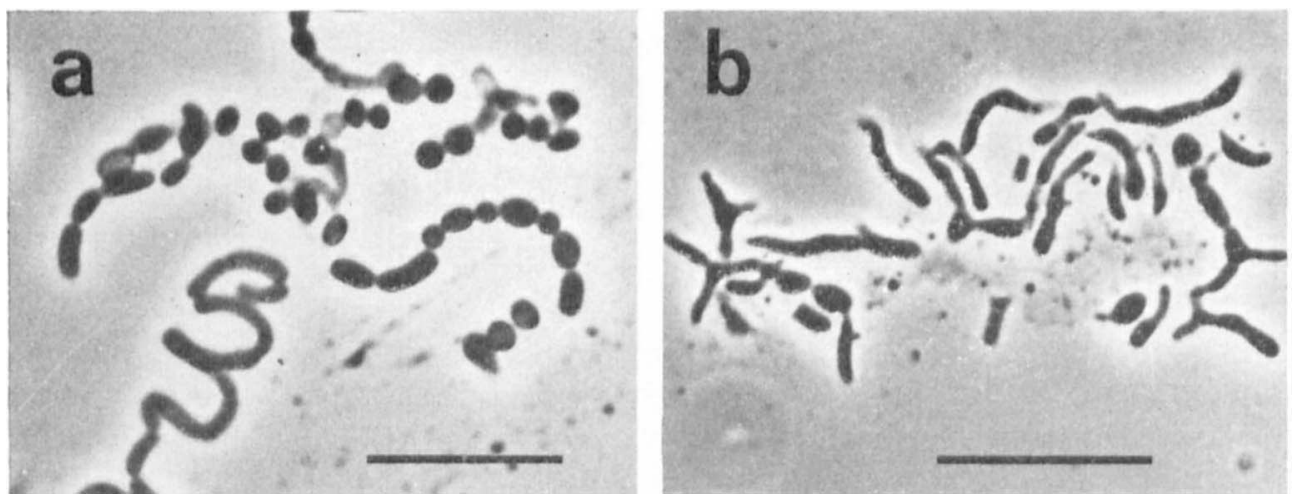

Fig. 3. Impression preparations from: $(a)$ wild type; $(b)$ bld A mutant (phase contrast). Bar markers represent I $\mu \mu \mathrm{m}$. The wild type shows chains of mature spores and coiled aerial mycelium with immature spores. The mutant (bld A39) shows branched mis-shapen hyphae with frequent septa. 

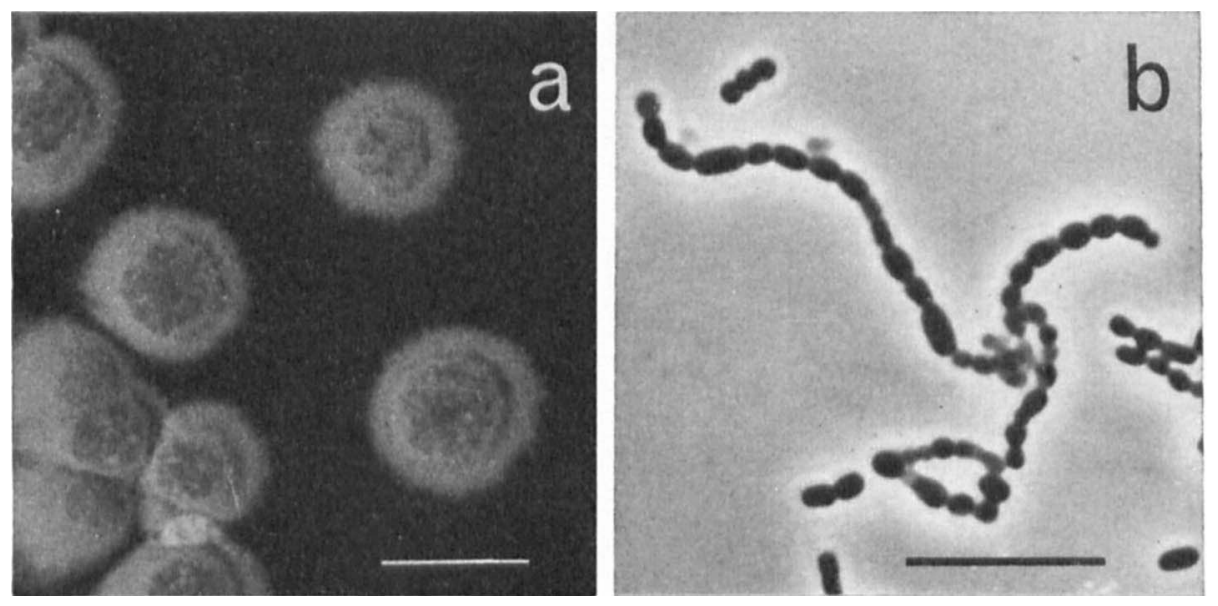

Fig. 4. Morphology of a bldA mutant grown on I \% mannitol as carbon source. (a) Colony morphology (bldA35) after 5 days at $30^{\circ} \mathrm{C}$. Bar marker represents $0.25 \mathrm{~cm}$. (b) Impression preparation (phase contrast) from colonies shown in $(a)$. The mutant shows chains of mature spores indistinguishable from those of the wild type (see Fig. $3 a$ ). Bar marker represents $10 \mu \mathrm{m}$.

carbon sources they produced aerial hyphae and spores (Fig. 4) which, in impression preparations, were indistinguishable from those of wild-type colonies (Fig. 4). Such 'corrected' strains did not, however, produce the pigments typical of wild-type $S$. coelicolor A3(2). Class I mutants remained bald when growing on CM regardless of the sugar or alcohol used to replace glucose.

Class 2 mutants were bald on all eight carbon sources. Growth on arabinose and mannitol was rather poor and sparse tufts of aerial hyphae were sometimes observed under these conditions. Impression preparations of such material indicated that spores were not produced and the aerial hyphae were similar in appearance to those seen in whiG mutants (Chater, 1972).

The class 3 mutant was bald on arabinose, glucose, mannose, cellobiose and glycerol, and produced aerial hyphae and spores on galactose, maltose and mannitol. Impression preparations of these aerial hyphae were indistinguishable from the wild type.

The wild type and the mutants grew well on MM with I or $0.1 \%$ glucose but grew only sparsely with 0.0 I $\%$ glucose. The phenotype of class I mutants was examined on MM with I \% mannitol further supplemented with glucose at I, 0.1 and 0.0 I $\%$. Colonies were completely bald with I or $0.1 \%$ glucose but sporulated when only $0.01 \%$ glucose was present.

Although neither the presence nor any effect of cyclic AMP has previously been demonstrated in Streptomycetes, the effects of glucose on bld colony morphology suggested that catabolite repression and cyclic AMP might be involved. However, both cyclic AMP and its $N^{6}, O^{2}$-dibutyryl derivative (added to $\mathrm{MM}$ at $5 \mathrm{mM}$ ) had no effect on the colony morphology of all three classes of bld mutants.

Streptomyces coelicolor A3(2) can utilize agar as a carbon source and will therefore grow on MM without added glucose. However, the parent strain $\mathrm{J} 650$, in which the bld mutants were isolated, carries the agaAr mutation (Fig. I) and cannot utilize agar as a carbon source (Hopwood et al., 1973). The metabolic lesion causing the aga phenotype is not known. The mutation does not apparently alter utilization of any sugars but does 
Table 2. Phenotypic characteristics of bld mutants

\begin{tabular}{|c|c|c|c|c|}
\hline \multirow[b]{2}{*}{ Character } & \multicolumn{4}{|c|}{ Morphological class } \\
\hline & Wild type & $\mathbf{I}$ & 2 & 3 \\
\hline Colony morphology* & Wild type & $\begin{array}{l}\text { Soft, frag- } \\
\text { menting } \\
\text { Sculptured } \\
\text { surface }\end{array}$ & $\begin{array}{l}\text { Hard, non- } \\
\text { fragmenting } \\
\text { Smooth } \\
\text { surface }\end{array}$ & $\begin{array}{c}\text { Soft frag- } \\
\text { menting } \\
\text { Smooth } \\
\text { surface }\end{array}$ \\
\hline Pigment production $\dagger$ & + & - & - & + \\
\hline $\begin{array}{l}\text { Antibiotic production } \ddagger \\
\text { Methylenomycin } \\
\text { Actinorhodin }\end{array}$ & $\begin{array}{l}+ \\
+\end{array}$ & $\overline{-}$ & $\overline{-}$ & $\begin{array}{l}+\S \\
+\end{array}$ \\
\hline $\begin{array}{l}\text { Morphology on differer } \\
\text { carbon sources\| }\end{array}$ & & & & \\
\hline Arabinose & + & + & $-p$ & - \\
\hline Galactose & + & + & - & + \\
\hline Glucose & + & - & - & - \\
\hline Mannose & + & - & - & - \\
\hline Cellobiose & + & - & - & - \\
\hline Maltose & + & + & - & + \\
\hline Glycerol & + & + & - & - \\
\hline Mannitol & + & + & $-p$ & + \\
\hline Representative bld loci & & bld $A$, bldD & bldB & bldC \\
\hline
\end{tabular}

prevent utilization of a wide range of amino acids as carbon sources, e.g. histidine, proline or arginine (Hopwood \& Merrick, unpublished results).

It was subsequently shown that the effects of the carbon source on the bld phenotype are the same in both $a g a A I$ and $a g a^{+}$strains and it is therefore probably not necessary to use an aga strain in order to classify a particular mutation with respect to its phenotype on different carbon sources.

\section{Antibiotic production by bld mutants}

Streptomyces coelicolor A3(2) produces two well-characterized antibiotics. Strains carrying the plasmid SCPI produce the antibiotic methylenomycin (Kirby, Wright \& Hopwood, I975; Wright \& Hopwood, I976a). Genes on SCPI code for a number of steps in the biosynthesis of this antibiotic and also for resistance to it (Kirby, 1976). SCPI ${ }^{-}$ strains do not produce methylenomycin and are sensitive to it. The second antibiotic, actinorhodin (Brockman et al., I966), is produced by both $\mathrm{SCPI}^{+}$and $\mathrm{SCPI}^{-}$strains (Wright \& Hopwood, 1976b) and mutations causing loss of actinorhodin production have been mapped to the chromosome (Wright \& Hopwood, $1976 b$; B. A. M. Rudd, personal communication). The parent strain $\mathrm{j650}$, from which most of the bld mutants were derived, carries the SCPI plasmid integrated into the chromosome. Such strains are referred to as $\mathrm{NF}$ and, like $\mathrm{SCPI}^{+}$strains, produce both methylenomycin and actinorhodin.

Bld mutants were tested for methylenomycin production by looking for antibiotic 
Table 3. Preliminary mapping of bld mutations

Examples are given of crosses with representatives from one of each of the phenotypic classes. Each bld mutant (inner circle) was crossed with strain I $_{5} \mathrm{I} 4$ and $c y s A^{+}$and cys $D^{+}$(indicated by triangles) were selected. The number against each allele in the diagrams gives the frequency of that allele among the recombinant progeny. In crosses with $\mathrm{J} 660$ and $\mathrm{J} 66 \mathrm{9}$ the segregation of the bld mutation with respect to two potentially closely linked markers is tabulated and the probability that segregation of each pair of markers is independent is given. In each case this probability is very low for one of the markers, indicating that the bld mutation is linked to this marker.

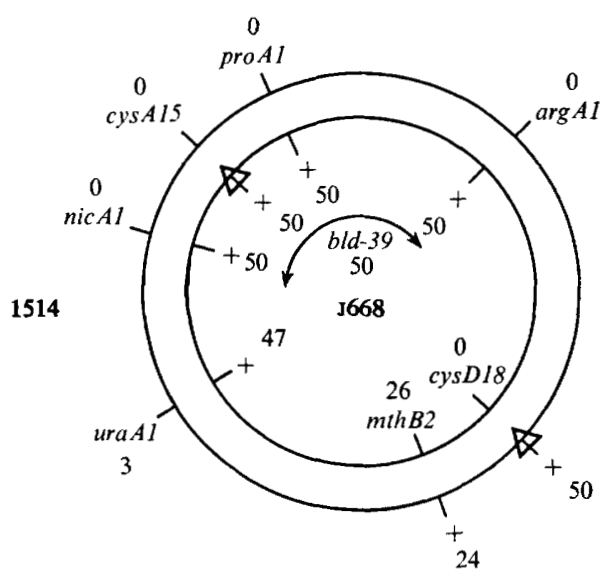

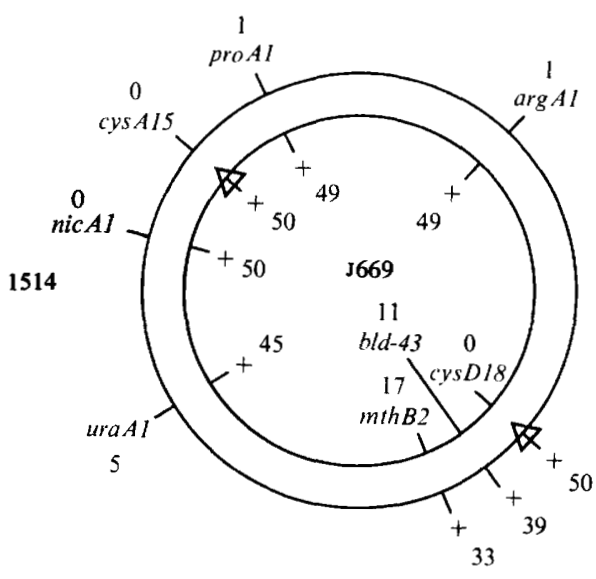

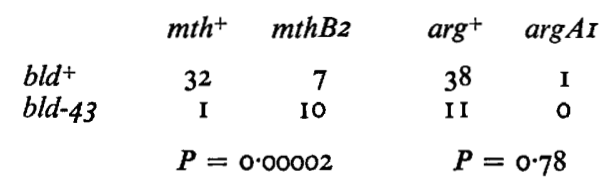

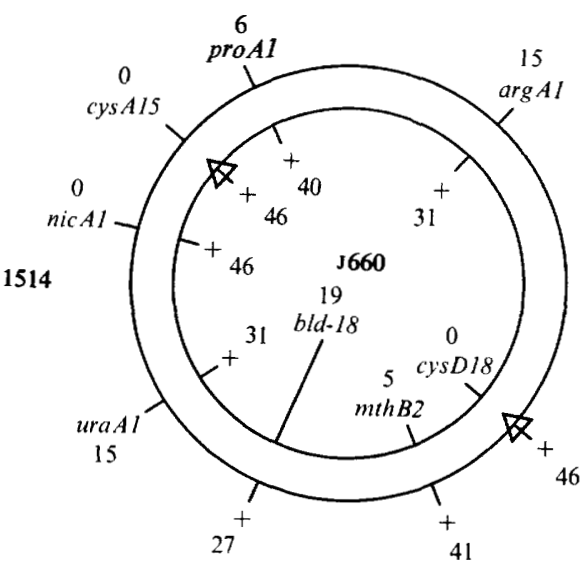

ura $^{+} \quad$ uraAI $\arg ^{+} \operatorname{argAI}$

$\begin{array}{lrcrr}\text { bld }^{+} & 12 & 15 & 15 & 12 \\ \text { bld-18 } & 19 & 0 & 16 & 3 \\ & P=0.00003 & & P=0.04\end{array}$

activity against $S$. coelicolor (strain I190), Bacillus cereus and Bacillus mycoides. Actinorhodin is a red/blue indicator pigment and therefore the production of this antibiotic was assessed visually, as well as by the activity of $\mathrm{SCPI}^{-}$bld strains against Staphylococcus aureus.

Class I mutants produced neither methylenomycin nor actinorhodin on $\mathrm{CM}$. SCPI ${ }^{+}$ wild-type strains produced small amounts of methylenomycin and normal amounts of 
Table 4. More precise location of bld-16, bld-35 and bld-39

The bld mutants were crossed with strain 949 and pro $A^{+} m t h B^{+}$(indicated by triangles) were selected. The number against each allele in the diagrams gives the frequency of that allele among the recombinant progeny. In each case the location of the bld mutation between $c y s A$ and nic $A$ is consistent with the gradient of allele frequencies.

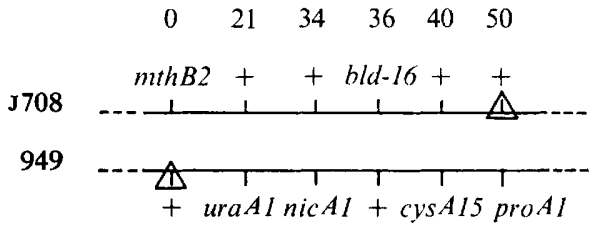

$\begin{array}{llllll}50 & 29 & 16 & 14 & 10 & 0\end{array}$

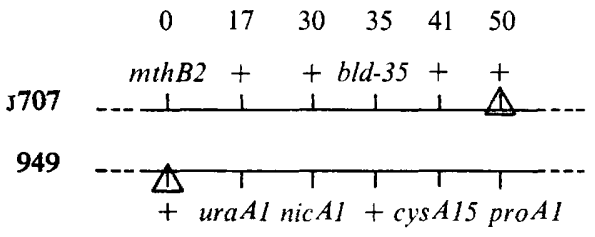

$\begin{array}{llllll}50 & 33 & 20 & 15 & 9 & 0\end{array}$

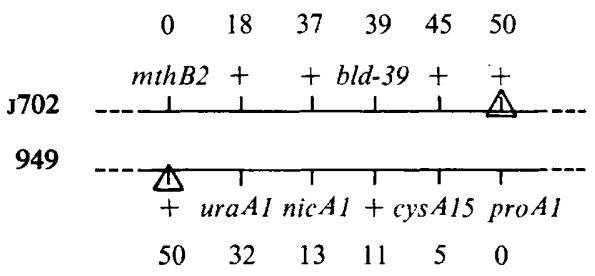

actinorhodin when grown on MM with I \% glucose or I \% mannitol. However, class I mutants grown on MM with $\mathrm{x} \%$ mannitol and showing a 'corrected' phenotype did not produce methylenomycin or actinorhodin (Chater, personal communication).

Class 2 mutants produced neither methylenomycin nor actinorhodin on CM.

The class 3 mutant produced actinorhodin and at least one other antibiotic which had a similar activity spectrum to methylenomycin. However, this mutant also showed antibiotic activity against Escherichia coli $\mathrm{KI} 2$ and Salmonella typhimurium LT2 whereas the wild-type $\mathrm{SCPI}^{+}$strains showed no antibiotic activity against these Gram-negative bacteria. When the class 3 mutation was transferred to an $\mathrm{SCPI}^{-}$strain only actinorhodin was produced, indicating that the other antibiotic activity was dependent upon the presence of SCPr. The phenotypic characteristics of each class of bld mutant are summarized in Table 2.

\section{Genetic analysis of the bld mutants}

In preliminary mapping studies, each mutant was crossed with the multiply-marked strain $15 \mathrm{I} 4$ and $c y s A^{+}$cys $D^{+}$recombinants were selected by plating on MM supplemented with all the growth requirements of the parent strains except cysteine. The genotypes of 
Table 5. Mapping of bldA39 with respect to adeC and agaA

Strain J668 was crossed with strain A200 using the selection indicated by triangles. Crossing-over in the upper arc of the map was due to the zygote structure in $\mathrm{NF} \times \mathrm{SCPI}^{-}$crosses. The allele frequencies obtained are shown in the diagram and the frequencies of the recombinant genotypes are tabulated.

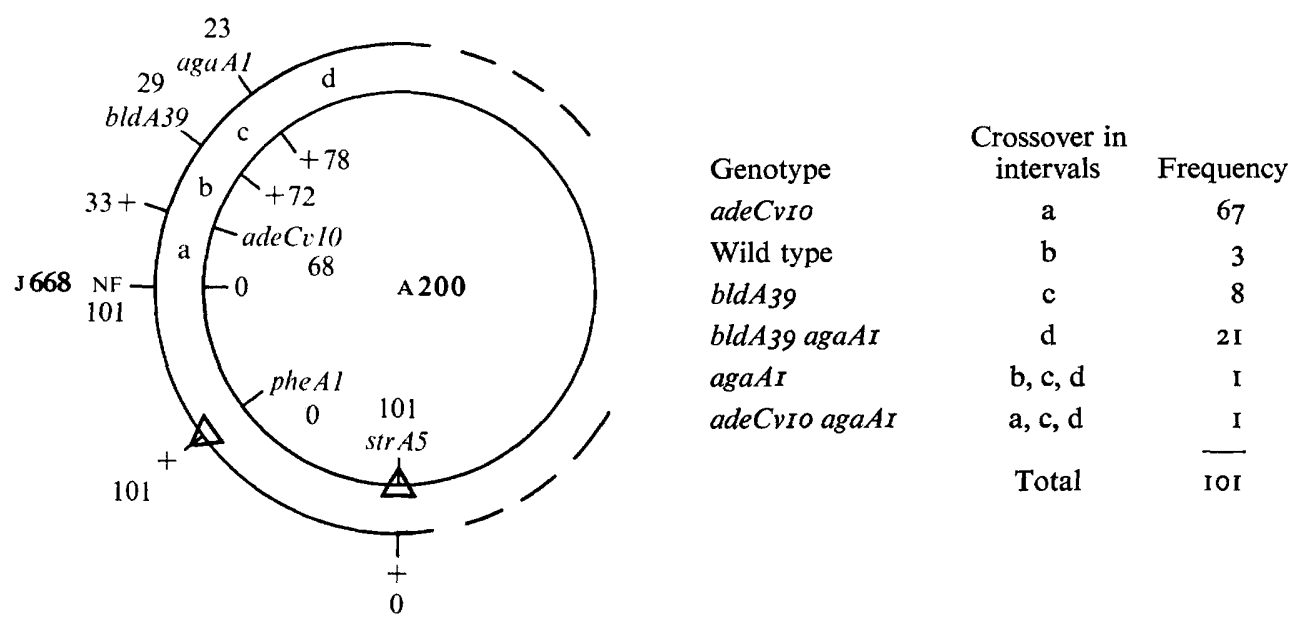

a sample of recombinants were then scored and the frequencies of each allele were inserted in a diagrammatic representation of the cross (Table 3). The frequency of the bld allele in the cross usually allowed two alternative locations, one in each of the arcs separating the selected markers. To decide between these locations, the segregation of the bld allele with respect to that of a potentially closely linked marker from each arc was tabulated. In most cases the segregation of the bld allele was dependent upon the segregation of one of the two chosen markers to which it was therefore linked, e.g. lack of independent segregation was found for bld-43 and $m t h B 2$ (Table 3).

For the three class I mutations (bld-16, bld-35, bld-39) originally mapped by this method, all or virtually all the recombinants were bld. These crosses therefore only indicated that the mutations were located between nicA and cys $D$ in the upper arc of the chromosome. An example of one such cross (with bld-39) is given in Table 3.

All the six class 2 mutations were located in the interval between $m t h B$ and cys $D$, e.g. bld-43 (Table 3), and the single class 3 mutation, bld-r8, was located in the uraA-strA interval (Table 3). With this information further crosses were devised to map each of the mutations more precisely.

\section{Class I mutants}

Bld recombinants carrying $m t h B z$ were selected from the preliminary crosses and crossed with strain 949. Selection was made for $m t h^{+}$pro $^{+}$recombinants and in each case the bld mutation mapped between nic $A$ and cys $A$ (Table 4). This location is the same as that found for bldAI (Hopwood, I967), which also has a class I phenotype. In order to examine whether all these class I mutations could be alleles of bldA, pairwise crosses were made between all four mutants in alternative coupling arrangements.

When crossing morphological mutants, recombinants carrying both mutations (double 
Table 6. Mapping of bldA35 with respect to whiB2I8

Strain $\mathrm{J} 733$ was crossed with strain $\mathrm{J} 200$ using the selection indicated by triangles. The allele frequencies obtained are shown in the diagram, and the frequencies of the recombinant genotypes are tabulated.

\begin{tabular}{|c|c|c|c|c|}
\hline & $\begin{array}{c}150122 \quad 150 \quad 41 \quad 320 \\
+\quad \text { uraAl }+ \text { bldA35 }+ \text { cys } A 15\end{array}$ & Genotype & $\begin{array}{l}\text { Crossover in } \\
\text { intervals }\end{array}$ & Frequency \\
\hline \multirow{5}{*}{ J 200} & $\Delta \triangle_{\mathrm{a}} \mathrm{d}_{\mathrm{b}} \mathrm{L}_{\mathrm{c}}$ & whiB218 & a & 109 \\
\hline & 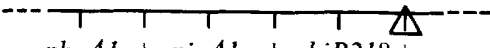 & bldA35 whiB218 & b & 9 \\
\hline & pheAI + nic $A 1+$ whiB218+ & bldA35 & c & 32 \\
\hline & & Wild type & $a, b, c$ & 0 \\
\hline & & & Total & 150 \\
\hline
\end{tabular}

mutants) are usually indistinguishable from one or both of the single mutants without further genetic analysis (Chater, 1975). As a result, ordering of the mutations is not possible by analysis of a single cross but relies on the relative frequencies of recovery of morphologically wild-type recombinants from crosses in alternative coupling arrangements. If the two mutations are in the same gene such recombinants will be rare in either coupling.

Bld nic $A I$ and bld cysAI 5 strains were constructed with each of the four class I mutations, bldAI, bld-16, bld-35 and bld-39. Each bld nicAI strain was then crossed to all the bld cys AI 5 strains and $n i c^{+}{ }^{c y} s^{+}$recombinants were selected. In no case were $b^{+}{ }^{+}$recombinants recovered. On the basis of these data, the three new bld mutations were assigned to the bldA locus (Fig. I). Unambiguous assignment of a bld mutation to a particular locus by complementation analysis is not possible at present in $S$. coelicolor as SCPI' plasmids are not available for the relevant sections of the genome (Hopwood \& Wright, 1976a).

In order to define the location of bldA more precisely, strain J668 was crossed to strain A200 and phe ${ }^{+}$strA5 recombinants were selected. This selection was made solely to exclude parental genotypes; crossing-over in the 9 o'clock to I2 o'clock region of the genome was due to the zygote structure in $\mathrm{NF} \times \mathrm{SCPI}^{-}$(UF) crosses (Hopwood et al., 1969). Data from this cross indicated that $b l d A$ was located between adeC and aga $A$ (Table 5).

Another mutation affecting development, whiB, is also located between nic $A$ and $c y s A$ (Chater, 1972) and it was therefore of interest to map bldA relative to whiB. Strain $\mathbf{J} 200$ was crossed with strain $\mathrm{J} 733$ and $\mathrm{cys}^{+}$nic $^{+}$phe ${ }^{+}$recombinants were selected (Table 6). When scoring recombinants from this cross, use was made of the ability to 'correct' the bld $A$ phenotype by growth on mannitol. By replicating master plates with glucose as carbon source to plates with mannitol as carbon source, it was possible to identify bldA35 $w h i^{+}$ and bldA35whiB218 recombinants and hence all four recombinant classes could be distinguished. The bldA35 mutation was located between whiB and nicA (Table 6). Recombinants of genotype bldA35 whiB2I 8 grown on mannitol had the normal whiB2I 8 phenotype described by Chater (1972) and when grown on glucose they showed a normal bldA phenotype.

A number of other bld mutants were isolated by Hopwood at the same time as bldAI but were not extensively analysed. Two of these mutations, bld-33 and bld-62, have been mapped between $c y s A$ and nic $A$ and gave no $b l d^{+}$recombinants when crossed in alternative coupling arrangements with $b l d A$ strains. They were found to have a class I phenotype 
Table 7. The location of bld-53

Strain I 169 was crossed with strain 949 and $h i s^{+}$str $A I$ recombinants were selected. The segregation of bld-53 with respect to $\arg A I$ and $p r o A I$ is tabulated and the contingency $\chi^{2}$ shows that the segregation of bld-53 is dependent upon that of $\arg A I$, and independent of proAI.

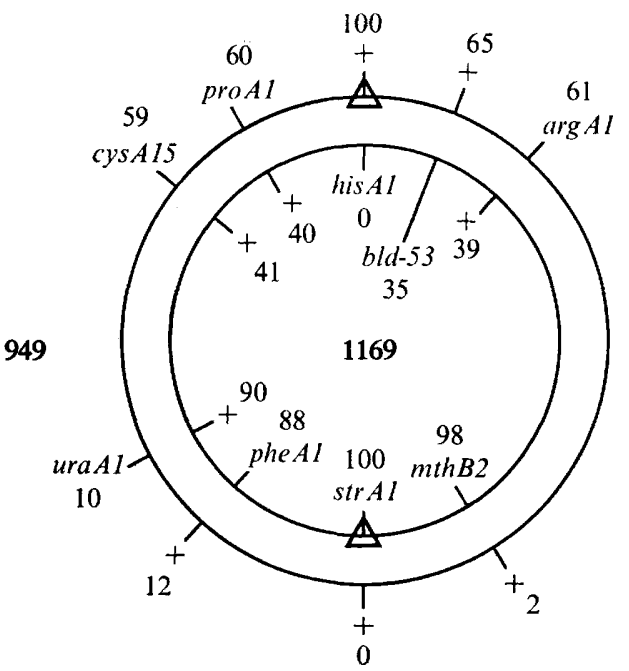

$\begin{array}{lccr} & \arg A^{+} & \arg A I & \text { Total } \\ \text { bld }{ }^{+} & 6 & 59 & 65 \\ \text { bld }-53 & \frac{33}{39} & \frac{2}{6 \mathrm{I}} & \frac{35}{100} \\ \text { Total } & P<0.00 \mathrm{I} & & \end{array}$

Table 8. Mapping of bldD53 with respect to whiA72

Strain I 169 was crossed with J93, and strain $\mathrm{J} 777$ was crossed with $\mathrm{JI} 2$ using the selection indicated by triangles. Replication to mannitol was used to distinguish bldD 53 and bldD 53 whiA72 recombinants. The allele frequencies obtained are shown in the diagram and the recombinant genotypes are tabulated.
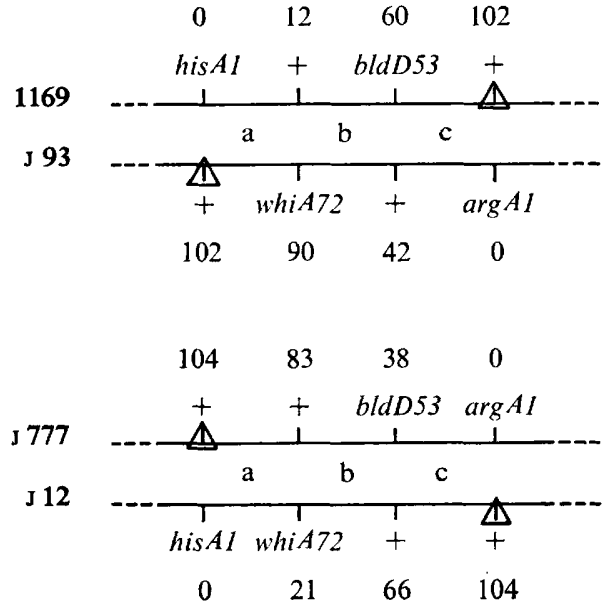

whiA72

Wild type

bldD 53

bldD53 whiA72
Crossover in intervals Frequency

bldD53

bldD53 whiA72

whiA72

Wild type

a

I 2

b

48

c

42

a, b, c

Total

IO2

$\begin{array}{ll}\mathrm{a} & \text { I8 } \\ \mathrm{b} & 48 \\ \mathrm{c} & 35\end{array}$

a, b, c 3

Total 
Table 9. Mapping of bldD53 with respect to ser $A \mathbf{I}$

Strain $\mathrm{J} 777$ was crossed with 948 using the selection indicated by triangles. The allele frequencies are shown in the diagram and the recombinant genotypes are tabulated.

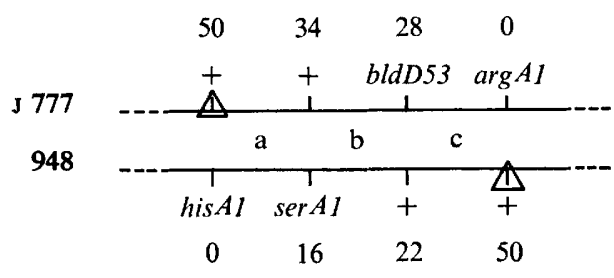

\section{Genotype \\ serAI}

Wild type

bldD53

bldD53 serAI

$\begin{array}{cc}\begin{array}{c}\text { Crossover in } \\ \text { intervals }\end{array} & \text { Frequency } \\ \text { a } & 9 \\ \text { b } & 13 \\ \text { c } & 2 \mathrm{I} \\ \text { a, b, c } & 7 \\ \text { Total } & 50\end{array}$

with the exception that they did not show the same pattern of morphological responses to different carbon sources as the other bldA mutations. A further mutation, bld-53, had an exact class $I$ phenotype and was therefore mapped to determine whether it was also a bldA mutation. The original mutant strain, I I 69, was crossed with strain 949 and is $^{+}$str $A I$ recombinants were selected. Analysis of the cross indicated that bld-53 was between his $A$ and $\arg A$ (Table 7) and it was therefore assigned to a new locus bldD (Fig. I). The unusual linkage relationship between uraA and phe $A$ in this cross is not understood, and the segregation of $\operatorname{nic} A$ was not scored.

Since whiA also lies between his $A$ and $\arg A$, bld-53 was mapped with respect to whiA. The cross was carried out in both coupling arrangements. Strain I 69 was crossed with $\mathrm{J} 93$ and strain $\mathrm{J} 777$ was crossed with $\mathrm{JI}_{2} ;$ arg $^{+}$his $^{+}$recombinants were selected. Replication to mannitol distinguished bldD53 and bldD53 whiA72 recombinants. The results from both crosses were consistent with a map location for bldD53 between whiA and $\arg A$ (Table 8).

Finally, bldD 53 was mapped with respect to $\operatorname{ser} A$ by crossing strain $\mathrm{J} 777$ with strain 948 and selecting his $^{+}$arg $^{+}$recombinants. This cross located bldD between $\operatorname{ser} A$ and $\arg A$ (Table 9).

\section{Class 2 mutants}

Preliminary mapping located all of the six class 2 mutations (bld-15, -17, -28, -43, -57 and -59 ) between $m t h B$ and $c y s D$. To refine this map location, $m t h B 2$ recombinant derivatives of each mutant were crossed with strain $\mathrm{JI} 92$ and $m t h^{+}$cys ${ }^{+}$recombinants were selected. In each case the bld mutation was located between leuB and $c y s D$. To determine whether all six mutations could be in the same gene, leu $B$ and $c y s D$ recombinant derivatives of each mutant were obtained. One of the mutants, bld-43, was then chosen as a tester strain and each of the other mutants was crossed to bld-43 in both coupling arrangements, with selection for $l e u^{+} c y s^{+}$recombinants. In all crosses the frequency of wild-type recombinants was lower than $0.01 \%$ and the six class 2 mutations were therefore all assigned to the bldB locus (Fig. 1). It should be emphasized that, although these mutations had similar phenotypes, they could be non-allelic.

Three whi loci (whiG, whiH, whiI) are located between cysD and leuB and crosses were designed to locate $b l d B$ with respect to these loci. A bldB43 strain (J669) carrying cys $D_{I} 8$ was crossed with leuB5 derivatives of whiG (JI 19), whiH (J49) and whiI (J86) respectively, and crosses in the alternative coupling arrangement were made using a leuB5 bldB43 
Table 10. Mapping of bldB43 with respect to whiG, whiH and whiI

Strain J669 was crossed with strains JI I9, J49 and J86 and strain J7I 4 was crossed with strains JI I 8, $\mathrm{J} 95$ and $\mathbf{\mathrm { J } 2 7}$. In all crosses $\mathrm{leu}^{+} \mathrm{cys}^{+}$recombinants were selected and their phenotypes were scored. The recombinant genotypes $b l d B$ and $b l d B$ whi were indistinguishable and so only minimum estimates (*) of the frequency of whi could be obtained.
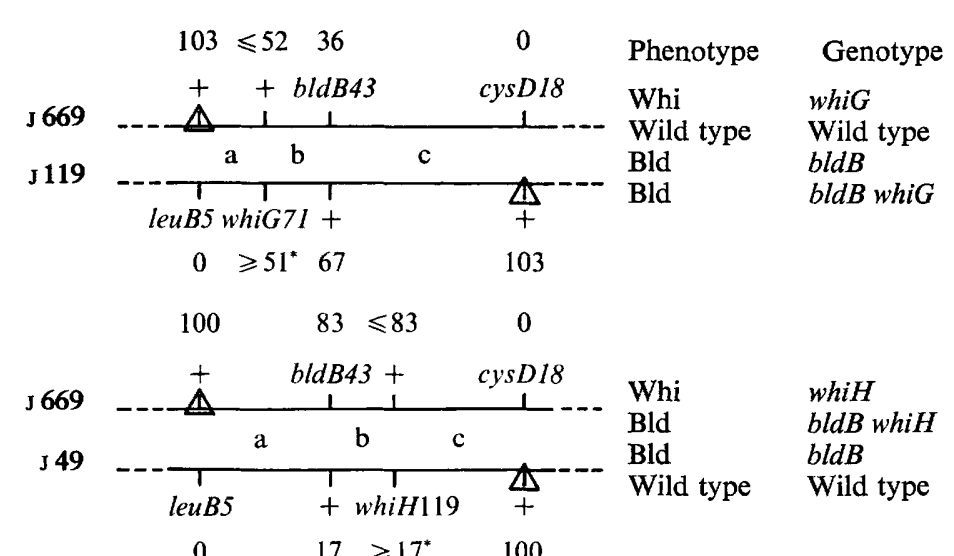

Whi

Bld

Wild type

0

$\geqslant 17^{*} \quad 100$

$\begin{array}{ll}56 & 24\end{array} 24 \quad 0$

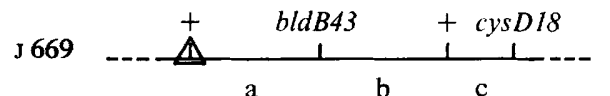

Whi

Bld

J 86

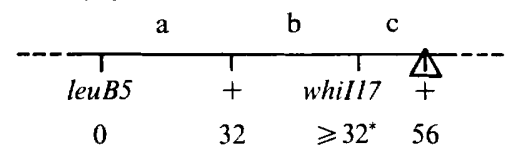

Bld

Wild type

$0 \leqslant 63 \quad 63$

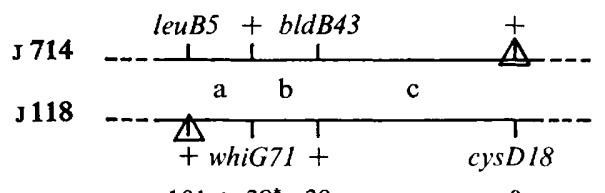

Bld

Bld

Whi

Wild type

$101 \geqslant 38^{*} 38$

0

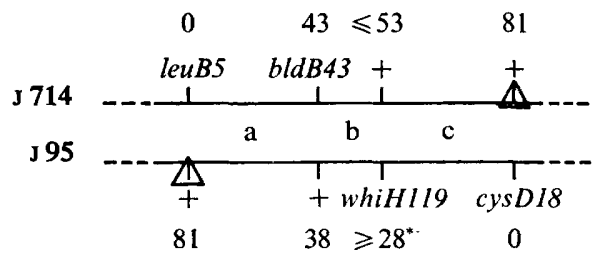

Bld

Bld

Wild type

Whi

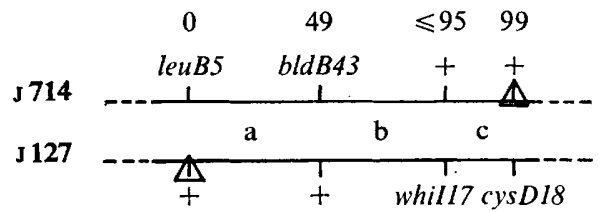

Bld

Bld

Wild type

Whi

99

$50 \geqslant 4^{*} \quad 0$
whiH

bldB whiH

bldB

Wild type

whil

bldB whil

bldB

Wild type

bldB

bldB whiG

whiG

Wild type

bldB

bldB whiH

Wild type

whiH

bldB

bldB whiI

Wild type

whil

\section{Crossover in}

intervals Frequency

$\begin{array}{cr}\begin{array}{c}\mathrm{a} \\ \mathrm{b} \\ \mathrm{c} \\ \mathrm{a}, \mathrm{b}, \mathrm{c}\end{array} & \begin{array}{r}5 \mathrm{I} \\ \mathrm{I} 6\end{array} \\ \text { Total } & 36\end{array}$

$\begin{array}{cr}\left.\begin{array}{c}\mathrm{a} \\ \mathrm{b} \\ \mathrm{c}\end{array}\right\} & \begin{array}{r}17 \\ \mathrm{a}, \mathrm{b}, \mathrm{c}\end{array} \\ \text { Total } & -13 \\ & -100\end{array}$

$\left.\begin{array}{l}\text { a } \\ \mathrm{b} \\ \mathrm{c}\end{array}\right\}$

32

a, b, c

24

Total

$\frac{0}{56}$

$\left.\begin{array}{l}\text { a } \\ b\end{array}\right\} \quad 63$

$\mathrm{a}, \mathrm{b}, \mathrm{c}$

Total IOI

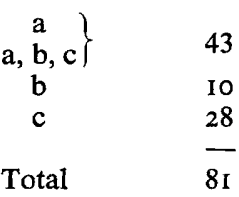

a

49

b 46

c 4

Total 99 
Table I I. More precise location of bld-18

Strain J74I was crossed with strain 285 using the selection indicated by triangles. The allele frequencies obtained are shown in the diagram and the recombinant genotypes are tabulated.

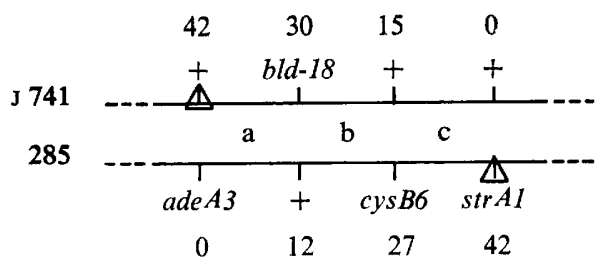

Genotype
cysB6
bld-I8 cysB6
bld-18
Wild type

$\begin{array}{cc}\begin{array}{c}\text { Crossover in } \\ \text { intervals }\end{array} & \text { Frequency } \\ \mathrm{a} & \text { 12 } \\ \mathrm{b} & \text { I5 } \\ \mathrm{c} & \mathrm{1} 5 \\ \mathrm{a}, \mathrm{b}, \mathrm{c} & \mathrm{0} \\ \text { Total } & -42\end{array}$

strain (J7I4) and cysDI8 derivatives of whiG (JI I8), whiH (J95) and whiI (JI27). In all six crosses $\mathrm{leu}^{+} \mathrm{cys}^{+}$recombinants were selected (Table Io).

Since $b l d B$ could not be induced to sporulate by changing the carbon source it was not possible to distinguish bldB43 and bldB43 whi recombinants. Interpretation of the crosses, except by genetic analysis of all the bld recombinants, therefore depended on the frequency of morphologically wild-type recombinants in each cross and on the assumption that $b l d B$ whi recombinants had a bld phenotype. The presence of bldB whi double mutants amongst the recombinants also meant that only minimum estimates of the frequency of whi could be obtained.

In crosses between $c y s D$ bldB and leuB whi strains (Table Io), wild-type recombinants could be obtained by a single crossover only if bldB were between whi and cys $D$. Such recombinants were obtained only when $b l d B$ was crossed with whiG, suggesting that bldB is between whiG and whiH. In crosses between leuB bldB and cysD whi strains (Table Io), wild-type recombinants could be produced by a single crossover only if $b l d B$ were between $l e u B$ and $w h i$. Wild-type recombinants were obtained in all three crosses but the low frequency ( $3 \%$ ) of such recombinants in the cross between bldB and whiG could be accounted for by multiple crossovers. The higher frequency of wild types in the other two crosses probably arose by single crossovers and hence these crosses are also consistent with a location of $b l d B$ between $w h i G$ and whiH. Assuming that this map location for bldB is correct, then in three crosses ( $6669 \times \mathrm{JI}_{19}, \mathrm{~J} 7 \mathrm{I} 4 \times \mathrm{J} 95$ and $\left.\mathrm{J} 7 \mathrm{I} 4 \times \mathrm{JI} 27\right)$ bld $B$ whi recombinants could arise only by triple crossovers and hence minimum estimates of the frequency of whi (assuming no triple crossovers) can be obtained (Table I0).

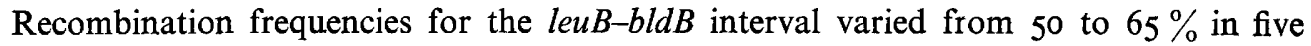
crosses, with a value of $17 \%$ in cross $\mathrm{J} 669 \times \mathrm{J} 49$. The precision of such estimates is, however, likely to be low owing to the multinucleate nature of most of the plating units and the possibility of selection against particular genotypes.

\section{Class 3 mutants}

In preliminary crosses $b l d-I 8$ was located between $u r a A$ and $s t r A$ and this map position was refined by crossing a $m t h B 2$ agaA $x$ bld- 18 recombinant (J74I) with strain 285 . Selection was made for ade ${ }^{+}$str $A I$ recombinants and bld-I8 was found to map between ade $A$ and cys $B$ (Table II). This location defined the fourth bld locus, bldC (Fig. I).

The relatively close linkage of $b l d C I 8$ to $c y s B$ prompted investigation of whether the $b^{+} d^{+}$allele of bldCI8 was carried by SCPI'-cysB (Hopwood \& Wright, I973). An SCPI'cys $B$, bldCr 8 strain was constructed and was found to have a bldC phenotype indicating 
either that the $b l d C^{+}$allele was not carried by the $\mathrm{SCPI}^{\prime}-$-cys $B$ plasmid or that the $b l d C^{+}$ allele was carried by $\mathrm{SCPI}^{\prime}-c y s B$ and $b l d C$ was dominant to $b l d C^{+}$. If the latter were the case then $b l d^{+}$homogenotes would be expected to arise in the same way as $c y s^{+}$or $c y s B$ homogenotes arise (Hopwood \& Wright, $1976 b$ ). Such $b l d^{+}$segregants were not observed and it was therefore concluded that the bldC gene is not carried by $\mathrm{SCPI}^{\prime}-c y s B$.

\section{DISCUSSION}

Four distinct map locations designated $b l d A, b l d B, b l d C$ and $b l d D$ have been identified and in each case mutations at the same location gave rise to a similar phenotype. Methods of selection for SCPI' plasmids carrying genes from any chosen region of the genome are not yet available and hence complementation tests between the bld mutations are not possible at present. Some of the clustered bld mutations may therefore be non-allelic as was noted for the whi mutations by Chater (1972).

The close linkage of three of the bld loci to whi loci is of particular interest. Mutations in bldA and bldD appear to produce an identical phenotype, as do mutations in whiA and $w h i B$, and this similarity is heightened by the fact that whiA is closely linked to bldD, and whiB is closely linked to bldA. Whether these phenotypic and linkage relationships could reflect biochemical similarities between the genes concerned is not known.

Studies on the cell-wall composition of bldA mutants have indicated that the wall composition differs from that found in $b l d^{+}$strains although the precise alteration involved has not yet been identified (unpublished results).

The bldB mutations have the phenotype which might be predicted for a mutation which allows only the production of vegetative mycelium, i.e. a hard leathery type of colony which cannot be induced to form aerial hyphae by changing the carbon source. Hence it is particularly interesting that $b l d B$ maps between $w h i G$ and whiH which are apparently mutations in very early steps in aerial mycelium formation (Chater, 1975).

There have been a number of reports of the possible involvement of plasmid-borne genes in aerial mycelium development (Okanishi, Ohta \& Umezawa, 1970; Kähler \& Noack, 1974; Redshaw et al., 1976). However, all the bld mutations mapped so far in $S$. coelicolor can be assigned unequivocally to map locations on the circular chromosome, although experiments using curing agents such as ethidium bromide have not been carried out.

Relatively few mutations have been investigated in this study and it is very likely that other bld loci await discovery. The mutants described were selected at random on the sole criterion that they should be completely bald and have a stable phenotype; other mutants were often found which produced sparse aerial mycelia and which sporulated poorly. Such phenotypes could frequently be due to trivial pleiotropic effects of mutations in genes not directly connected with colony development but, even if they represent mutations in important loci, their analysis would be extremely difficult owing to problems in classifying recombinants.

Asporogenous variants have been described in a number of Streptomyces species (Appleby, 1948; Williams \& McCoy, 1953; Perlman, Greenfield \& O’Brien, 1954; Nakamura, I965; Nikitina \& Kalakoutskii, I $973 a, b$ ). In all cases these variants arose as secondary colonies or sectors during serial transfer and therefore their genetic origins are obscure. The lack of a genetic system in any of the organisms prevented genetic analysis of the variants and hence one cannot exclude the possibility that they are due to multiple 
mutations. By contrast all the bld mutants described here have been shown to be due to single mutations.

The variants of $S$. griseus described by Williams \& McCoy (1953), Perlman et al. (1954) and Nakamura (1965) did not produce the antibiotic, streptomycin, produced by the parent. The asporogenous strains described by Nakamura (1965) also fragmented considerably after 5 or 6 days' growth and failed to produce both the pigments and the characteristic earthy odour produced by the parent strain. Similarly, some of the asporogenous variants described by Nikitina \& Kalakoutskii $(1973 a, b)$ showed a tendency to fragment and were referred to as pasty in texture whilst others were described as having a leathery texture. In all cases the variants produced similar quantities of antibiotic to the parent strains. All of these variants appear to share some characteristics of the bld mutants described here and might possibly result from similar changes in metabolism.

The pleiotropic effects of all the bld mutations on the production of both antibiotics and pigments suggests that these characteristics may have regulatory links with certain developmental processes, although mutations in characters such as cell-membrane composition might cause these pleiotropic effects by disrupting the function of certain membrane-bound enzymes.

The morphological correction of bldA, bldC and bldD mutants grown on certain carbon sources is particularly useful when characterizing bld whi double mutants. It also suggests that the lesions causing these bld phenotypes could be in some aspect of the organism's carbon metabolism. The lack of correction when bldA mutants are grown on a mixture of glucose and mannitol could be due to carbon catabolite repression but seems more likely to be attributable to preferential utilization of glucose. Comparable effects of carbon source on morphology were observed by Nikitina \& Kalakoutskii (1973 $b$ ) in asporogenous variants of $S$. albocyaneus and $S$. anthocyaneus. In both cases the variants were bald on fructose-containing minimal medium but produced aerial mycelium on minimal media with glucose or glycerol as carbon source. Nikitina \& Kalakoutskii (I973a) also showed that the emergence of asporogenous variants of $S$. roseoflavus var. roseofungini grown on fructose was suppressed by addition of very low levels of mannitol.

This study of bld mutants in S. coelicolor A3(2) has considerably extended our knowledge of developmental mutants in this organism but further investigation of the mutants described here and isolation of more bld mutants (possibly denoting new loci) is now required. Aspects such as the phenotypic suppression of some bld phenotypes by changes in the carbon sources, and the pleiotropic effects of bld mutations on antibiotic production may well be areas in which further study could be rewarding.

I am grateful to David Hopwood and Keith Chater for their suggestions during this work and for helpful comments on the manuscript. I should like to thank the Directors of Pfizer Ltd for a postdoctoral fellowship.

\section{REFERENCES}

APPLEBY, J. C. (1948). An asporogenous variant of Streptomyces griseus. Journal of General Microbiology 2, 80-82.

Brockman, H., Zeeck, A., van der Merne, K. \& Müller, W. (1966). Die Konstitution des Actinorhodins. Justus Liebigs Annalen der Chemie 698, 209-229.

CHATER, K. F. (1972). A morphological and genetic mapping study of white colony mutants of Streptomyces coelicolor. Journal of General Microbiology 72, 9-28.

ChATER, K. F. (1975). Construction and phenotypes of double sporulation deficient mutants in Streptomyces coelicolor A3(2). Journal of General Microbiology 87, 312-325. 
Chater, K. F. \& Hopwood, D. A. (1973). Differentiation in Actinomycetes. Symposia of the Society for General Microbiology 23, 143-160.

ChATER, K.F. \& MERRICK, M.J.(1976). Approaches to the study of differentiation in Streptomyces coelicolor A3(2). In Second International Symposium on the Genetics of Industrial Microorganisms, pp. 583-593. Edited by K. D. Macdonald. London: Academic Press.

Delić, V., Hopwood, D. A. \& FrIEND, E. J. (1970). Mutagenesis by $N$-methyl- $N$ '-nitro- $N$-nitrosoguanidine (NTG) in Streptomyces coelicolor. Mutation Research 9, 167-182.

Hopwood, D. A. (1959). Linkage and the mechanism of recombination in Streptomyces coelicolor. Annals of the New York Academy of Sciences 8r, 887-898.

Hopwood, D. A. (1967). Genetic analysis and genome structure in Streptomyces coelicolor. Bacteriological Reviews 31, 373-403.

Hopwood, D. A. \& WRIGHT, H. M. (1973). A plasmid of Streptomyces coelicolor carrying a chromosomal locus and its inter-specific transfer. Journal of General Microbiology 79, 331-342.

Hopwood, D. A. \& WRIGHT, H. M. (I976a). Interactions of the plasmid SCPI with the chromosome of Streptomyces coelicolor A3(2). In Second International Symposium on the Genetics of Industrial Microorganisms, pp. 607-6r9. Edited by K. D. Macdonald. London: Academic Press.

Hopwood, D. A. \& WRIGHT, H. M. $(1976 b)$. Genetic studies on SCPI-prime strains of Streptomyces coelicolor A3(2). Journal of General Microbiology 95, I07-120.

Hopwood, D. A., Harold, R. J., Vivian, A. \& Ferguson, H. M. (I969). A new kind of fertility variant in Streptomyces coelicolor. Genetics $62,46 \mathrm{I}-477$.

Hopwood, D. A., Chater, K. F., Dowding, J. E. \& Vivian, A. (I973). Advances in Streptomyces coelicolor genetics. Bacteriological Reviews 37, 37I-405.

KäHLER, R. \& NoACK, D. (I974). Action of acridine orange and ethidium bromide on growth and antibiotic activity of Streptomyces hygroscopicus JA6599. Zeitschrift für allgemeine Mikrobiologie 14, 529-533.

KIRBY, R. (1976). Genetic studies on Streptomyces coelicolor Plasmid One. Ph.D. thesis, University of East Anglia.

KIRBY, R., Wright, L. F. \& Hopwood, D. A. (I975). Plasmid-determined antibiotic synthesis and resistance in Streptomyces coelicolor. Nature, London 254, $265-267$.

Nakamura, L. (1965). Asporogeny in Streptomyces. Dissertation Abstracts 25, 6896.

Nikitina, E. T. \& KalakoutSkII, L. V. (I973a). Cultivation conditions and expression of 'fructose effect' in Actinomyces roseoflavus var. roseofungini. Journal of General and Applied Microbiology 19, 85-96.

Nikitina, E. T. \& KaLAKOUTSKII, L. V. (I973 $b$ ). Actinomycete variants with impaired differentiation: production in high yield, recognition and phenotypic characterisation in seven species. Journal of Applied Bacteriology 36, 599-6I 8.

Okanishi, M., OHta, T. \& UMezawa, H. (1970). Possible control of formation of aerial mycelium and antibiotic production in Streptomyces by episomic factors. Journal of Antibiotics 23, 45-47.

Perlman, D., Greenfield, R. B. \& O'Brien, E. (I954). Degeneration of a Streptomyces griseus mutant on repeated transfer. Applied Microbiology 2, 199-202.

Redshaw, P. A., McCann, P. A., Sankaran, L. \& Pogell, B. M. (1976). Control of differentiation in Streptomycetes: Involvement of extrachromosomal deoxyribonucleic acid and glucose repression in aerial mycelia development. Journal of Bacteriology 125, 698-705.

TownSEND, M. E., WRIGHT, H. M. \& Hopwood, D. A. (197I). Efficient mutagenesis by near ultraviolet light in the presence of 8-methoxypsoralen in Streptomyces. Journal of Applied Bacteriology 34, 799801 .

Williams, A. M. \& McCoy, E. (1953). Degeneration and regeneration of Streptomyces griseus. Applied Microbiology I, 307-313.

WRIGHT, L. F. \& HoPwOoD, D. A. (I976a). Identification of the antibiotic determined by the SCPI plasmid of Streptomyces coelicolor A3(2). Journal of General Microbiology 95, 96-106.

Wright, L. F. \& HoPwood, D. A. $(1976 b)$. Actinorhodin is a chromosomally-determined antibiotic in Streptomyces coelicolor A3(2). Journal of General Microbiology 96, 289-297. 